\title{
Composition and Quality of Mule Deer Diets on Pinyon-Juniper Winter Range, Colorado
}

\author{
RICHARD M. BARTMANN
}

\begin{abstract}
Estimates of botanical composition and nutritional quality of mule deer diets on pinyon-juniper winter range in Piceance Basin, Colorado, were based on forage selections of 8 tame animals. Diets contained nearly all browse in early winter, but browse content decreased and forbs increased as winter progressed until April when consumption of new grass growth increased sharply. Dietary crude protein levels were marginally adequate for body maintenance during much of the winter. Levels of dietary in vitro digestible dry matter were inadequate. Browse was considered critical to winter survival of deer in Piceance Basin because it was the most available forage in deep snow. Also, its nutritional value was comparable or better than that of forbs and grasses selected by deer except in April when new plant growth was available. In spite of large variation in diet compositions, deer apparently selected forage mixes to maintain a consistent, although inadequate, diet quality through the critical wintering period.
\end{abstract}

Piceance Basin in northwest Colorado is winter range for a large segment of the White River mule deer (Odocoileus hemionus) population. Impending oil shale development and associated problems of spent shale disposal and reclamation of disturbed areas have created widespread concern for the future welfare of deer in the basin. This future is clouded by the inability to predict how oil shale development will proceed and the eventual course and success of reclamation efforts. A current dilemma involves mitigation for areas already, or soon to be, removed from wildlife use by development. Mitigation is often viewed as some form of habitat improvement. For most wildlife species, particularly mule deer, what should be improved, how it can be improved, or even if improvement is practical or needed are elusive questions. Therefore, knowledge is needed of how deer utilize present winter habitats for both food and cover. This study examines patterns of food use by mule deer in undisturbed habitats. Objectives were to identify major species in winter-long deer diets to estimate quality of those diets on native pinyon-juniper (Pinus edulis-Juniperus osteosperma) range in Piceance Basin.

\section{Study Area}

Piceance Basin includes primarily Piceance Creek and Yellow Creek drainages; an area encompassing about $2,600 \mathrm{~km}^{2}$. Pinyon and juniper are the dominant vegetation on most upland areas to about $2,300 \mathrm{~m}$ although extensions occur to near $2,500 \mathrm{~m}$. Major shrubs include big sagebrush (Artemisia tridentata), Utah serviceberry (Amelanchier utahensis), true mountain mahogany (Cercocarpus montanus), antelope bitterbrush (Purshia tridentata), mountain snowberry (Symphoricarpos oreophilus), low and rubber rabbitbrushes (Chrysothamnus viscidiflorus, C. nauseo-

\footnotetext{
Author is a wildlife researcher, Colorado Division of Wildlife, 317 West Prospect, Fort Collins, Colorado 80526.

Funding was provided by Colorado Federal Aid in Wildlife Restoration Project $W-38-R$ and $W-126-R$.

Appreciation is extended to the numerous persons that assisted with raising and caring for the tame deer, collection of field data, and laboratory and data analyses. The manuscript was reviewed by D.L. Baker, L.H. Carpenter, D.J. Freddy, N.T. Hobbs, and T.M. Pojar.

Manuscript received Oct. 4, 1982.
}

sus), and Gambel oak (Quercus gambelii). Dense stands of sagebrush also occur along and at the heads of many intermittent drainages. In extensive areas of the western basin, sagebrush is the dominant and sometimes the only shrub under pinyon-juniper canopy and in broad open parklands common on western mesas. Common forbs within the pinyon-juniper type include tailcup lupine (Lupinus caudatus), Nuttall goldenweed (Haplopappus nuttallii), cryptantha (Cryptantha sericea), Louisiana sagewort (Artemisia ludoviciana), Hood's phlox (Phlox hoodii) sulphur criogonum (Eriogonum umbellatum), and scarlet globemallow (Sphaeralcea coccinea). Common grasses are Indian ricegrass (Oryzopsis hymenoides), bluegrasses (Poa spp.), bluebunch and western wheatgrass (Agropyron spicatum, A. smithii), needle and thread (Stipa comata), and sedges (Carex spp.). At higher elevations, the pinyon-juniper type yields to a mountain shrub community containing many of the same shrub, forb, and grass species found at lower elevations, but usually at higher densities due to increased precipitation. Grass hay is produced in irrigated meadows along Piceance Creek and other drainages while sagebrush, black greasewood (Sarcobatus vermiculatus), and rubber rabbitbrush dominate unirrigated sections. Additional descriptions of the basin were given by Terwilliger et al. (1974), Voorhies (1974), and Tiedeman and Terwilliger (1978).

Deer begin migrating to winter range in October. While deer can be found throughout the winter range at this time, most remain in the mountain shrub type and upper elevation pinyon-juniper. Deepening snow in November and December forces deer to lower wintering areas with north aspects preferred. A $1,722-\mathrm{km}^{2}$ winter range was previously defined for the mid-winter (January) period and included most areas below about 2,300 m (Bartmann and Steinert 1981). As winter progresses, the upper limit of deer use drops closer to 2,100 $\mathrm{m}$ and deer shift to south aspects. Migration back to summer range begins in April with most deer gone from winter range by early May.

\section{Methods}

\section{Diet Composition}

Forage choices of tame mule deer were observed to estimate botanical composition of winter diets. Emphasis in treatment of experimental animals prior to and during the study was on maximum familiarity with, and dependence on native forages. Rearing and maintenance procedures from birth to 16 months of age were reported by Bartmann and Carpenter (1982). From age 16 months to the end of the study, when the deer were 34 months old, they continued to live year around in several pastures of 34 to 58 ha on pinyon-juniper range. They were removed only for foraging trials and for a month each fall during deer hunting season. While in the pastures, each deer was given about one-third $\mathrm{kg}$ of alfalfa hay and one-third $\mathrm{kg}$ of concentrate/day except from May to September when only concentrate was provided 3 times/week. Regelin et al. (1976) and Bartmann et al. (1982) found artificial feed had little or no effect on forage choices by tame deer. From December to March each year, rations were often shared with 7-12 wild deer that managed to enter the pasture during winter so quantities tame deer consumed were less than provided. This nominal feeding enabled 
checking deer numbers and condition and maintained a response to artificial feed necessary for controlling deer during foraging trials. Water was provided only when snow was unavailable.

lame deer foraging trials were conducted in 2 areas of Piceance Basin to include variation in vegetation conditions. The first area, in the northwest part of the basin, was a pinyon-juniper-mixed shrub habitat (Area 1) and the second, in the southwest part, a pinyon-juniper-sagebrush habitat (Area 2). Many of the same species occurred in both areas so differences were mainly in composition, particularly of shrubs. Distribution of shrubs, except sagebrush, was more restricted in the pinyon-juniper-sagebrush habitat. Deer densities were also lower than in the pinyon-junipermixed shrub habitat (Bartmann and Steinert 1981).

Each study area was divided into high, middle, and low elevation winter ranges. Five sampling periods (no January trials) in 1978-79 (Year 1) and 6 in 1979-80 (Year 2) were structured to approximate general patterns of use of these ranges by wild deer. High elevation winter range was sampled in October, middle elevation range in November, January, and April, and low elevation range in February and March. Sampling procedures were the same on both study areas each year but sequence of areas was alternated for the start of trials each sampling period.

Foraging trials were conducted on 3 sites/study area each sampling period. Random selection of sites was impractical as choices were restricted to those with signs of current deer use and with vehicular access, both of which could not alway be determined until immediately prior to foraging trials. Sites were located $1-3 \mathrm{~km}$ apart and situated to expose deer to a variety of vegetation and terrain conditions. Recurrent use by wild deer allowed using the same sites during the same sampling periods both years.

Foraging trials were conducted with 2 observers and 8 deer. A trial involved 1 person recording the number of bites and plant parts taken of each species by 1 deer during a $60-90$ minute period. Observers were rotated among deer, and deer among daily observation times. All 8 deer were observed daily for 3 days for a total of 24 trials/study area (16 trials/area in April 1980). Ample water and the usual ration of alfalfa hay and concentrate were provided each evening.

Deer were transported to the first site the day before starting trials and allowed several foraging excursions in the area. At night, and when not foraging during the day, they were kept in a temporary $8 \times 10-\mathrm{m}$ pen. The first morning, 4 deer were released from the pen and allowed to roam at will. Forage choices were recorded for 2 of them. Direction of travel was influenced by observers only if the deer started following trails or roads, or returned to the pen before they finished feeding. When the 2 simultaneous trials were completed, deer were returned to the pen, the other 4 released, and 2 again observed for data collection. Trials continued in the afternoon with the remaining 2 deer of each group observed. After all deer were observed, the group was walked to the next site, penned overnight, and another set of trials run the next day. After trials at the third site, deer were transported to the other study area and the process repeated. Deer were then returned to the holding pasture until the next sampling period.

The first year, the 8 deer consisted of 4 females and 4 male castrates. All were 15 months old when the study began. Two females died the following summer and were replaced by 2 male castrates. One replacement had been along on all trials the first year and the other, although raised with the group, had been used in a pen study from October to April the first winter. Loss of both replacements in late March 1980 left 6 deer for the last sampling period in April 1980.

A diet for an individual deer was the proportions of all forages, on a dry-weight basis, eaten on a study area during a sampling period. Individual diets were averaged across all deer to obtain a composite diet. Conversion of bites to a dry-weight basis reduced bias due to large bite-weight differences between some species. From 25-50 hand-picked "bites" of major species that composed $\geq 2 \%$ of the group's total bites on each study area were collected during each sampling period in Year 1 . Samples were oven-dried at $100^{\circ} \mathrm{C}$ for 48 hours, weighed to the nearest $0.1 \mathrm{~g}$, and divided by the total "bites" to obtain an average weight/bite. Bite-weight for other species was estimated by reference to values for species of similar form that were collected. In Year 2, bite-weight sampling was done only for major species not previously collected and also when bite size for a species was suspected to differ appreciably from Year 1 .

\section{Diet Quality}

For nutritional analyses, at least $50 \mathrm{~g}$ (green weight) of each major forage species were collected from the same plant and plant parts selected by the deer, or from adjacent ones if most or all of a plant was eaten. Forage samples were collected by the 2 observers, or by a third person, concurrent with foraging trials. Any additional collections needed were made immediately after completion of trials on an area. Samples were obtained over all 3 sites on a study area to include variability in growing conditions. Samples were placed in plastic bags and frozen. Later, they were dried at $60^{\circ}$ $\mathrm{C}$ for $\mathbf{4 8}$ hours, ground, and stored in glass jars. Methods for measuring crude protein $(\mathrm{CP})(\mathrm{Kjeldahl} \mathrm{N} \times 6.25)$ were from A.O.A.C. (1965). In vitro digestible dry matter (IVDDM) was estimated from triplicate $0.1-\mathrm{g}$ samples with methods described by Tilley and Terry (1963) and Pearson (1970). Rumen inoculum was from a Holstein cow maintained on grass hay, a diet considered reasonably comparable in quality to that afforded deer in winter. A separate in vitro run was made for each year's samples. Nutritional values of composite diets were derived by first adjusting proportions of major species to sum to $100 \%$. The CP and IVDDM percentages for each species were then weighted by summing the products of these adjusted proportions times their respective forage quality values (Hobbs et al. 1979). Differences in proportions of forage classes and CP and IVDDM content of diets between areas and years were assessed with paired or unpaired $t$ tests as appropriate.

Notes were made of relative species availability (low, medium, or high) on each study a rea during each sampling period. Such visual ratings, although subjective, took into account changes due to phenology and snow on each site and considered only locations used by tame deer. They were therefore considered most practical, particularly when the effort and imprecision associated with other availability estimate methods were considered and in light of the problems with assigning preference ratings discussed by Hobbs and Bowden (1982). The term browse includes both tree and shrub material, forbs include lower plant forms, and grass includes grasslike species.

\section{Results}

\section{Diet Composition}

Tame deer were observed for 685 hours and took over 641,000 bites from 139 plant species (Tables 1 and 2). From 18-59 plant species $(\bar{x}=46)$ made up each of 22 composite diets and 4-17 species were $\geq 2 \%$ of any one of these diets. Greatest variety occurred in late winter and spring diets on both areas. Forage selections varied considerably among deer as precision better than $\pm 20 \%$ of $\bar{x}$ at the $90 \%$ confidence level was seldom attained in percentage estimates for individual species in the 22 diets. For forage classes, this level of precision was attained or exceeded most consistently with browse. High variability in forage selections was previously shown for tame deer by Bartmann and Carpenter (1982) and was also evident in bite-count data for tame elk (Cervus elaphus) (Hobbs et al. 1981, Baker and Hobbs 1982).

Browse was the main diet component during all foraging periods except April when new growth of forbs and grasses was avidly sought. Browse use peaked in November and declined through winter and spring. Forbs assumed increasing importance over this same period until April when deer ate more grass. During early October, leaves of deciduous shrubs were turning color but most still persisted and were favored over twigs. For oak, preference of leaves over stems continued throughout winter. Forbs such as toadflax (Comandra umbellata), Spearleaf eriogonum (Eriogo- 
Table 1. Percentages of major forage species in tame mule deer diets on pinyon-juniper-mixed shrub winter range in Piceance Basin, Colorado, 1978-79 and 1979-80.'

\begin{tabular}{|c|c|c|c|c|c|c|c|c|c|c|c|c|c|}
\hline \multirow[b]{2}{*}{ Species } & \multirow[b]{2}{*}{ Year } & \multicolumn{2}{|c|}{ October } & \multicolumn{2}{|c|}{ November } & \multicolumn{2}{|c|}{ January } & \multicolumn{2}{|c|}{ February } & \multicolumn{2}{|c|}{ March } & \multicolumn{2}{|c|}{ April } \\
\hline & & $x$ & $\mathrm{SE}$ & $\bar{x}$ & $\mathrm{SE}$ & $\bar{x}$ & SE & $\bar{x}$ & SE & $\bar{x}$ & SE & $\bar{x}$ & $\widehat{S E}$ \\
\hline $\begin{array}{l}\text { Trees and Shrubs } \\
\text { Amelanchier utahensis }\end{array}$ & $\begin{array}{l}1979 \\
1980\end{array}$ & $\begin{array}{l}34 \\
13\end{array}$ & $\begin{array}{l}3 \\
2\end{array}$ & $\begin{array}{l}27 \\
20\end{array}$ & $\begin{array}{l}3 \\
2\end{array}$ & 18 & 3 & $\begin{array}{l}10 \\
10\end{array}$ & $\begin{array}{l}2 \\
3\end{array}$ & $\begin{array}{l}15 \\
12\end{array}$ & $\begin{array}{l}3 \\
2\end{array}$ & $\begin{array}{l}13 \\
20\end{array}$ & $\begin{array}{l}2 \\
7\end{array}$ \\
\hline Artemisia tridentata & $\begin{array}{l}1979 \\
1980\end{array}$ & $\begin{array}{l}\mathrm{tr} \\
\mathrm{tr}\end{array}$ & & $\begin{array}{l}2 \\
2\end{array}$ & $\begin{array}{l}1 \\
0.5\end{array}$ & 1 & 0.2 & $\begin{array}{l}2 \\
3\end{array}$ & $\begin{array}{l}1 \\
1\end{array}$ & $\begin{array}{r}6 \\
11\end{array}$ & $\begin{array}{l}1 \\
3\end{array}$ & $\begin{array}{l}3 \\
7\end{array}$ & $\begin{array}{l}1 \\
1\end{array}$ \\
\hline Atriplex confertifolia & $\begin{array}{l}1979 \\
1980\end{array}$ & & & & & 1 & 1 & $\begin{array}{l}1 \\
4\end{array}$ & $\begin{array}{l}0.5 \\
1\end{array}$ & $\begin{array}{l}1 \\
2\end{array}$ & $\begin{array}{l}1 \\
1\end{array}$ & & \\
\hline Cercocarpus montanus & $\begin{array}{l}1979 \\
1980\end{array}$ & $\begin{array}{l}21 \\
44\end{array}$ & $\begin{array}{l}2 \\
4\end{array}$ & $\begin{array}{l}30 \\
20\end{array}$ & $\begin{array}{l}4 \\
2\end{array}$ & 3 & 1 & $\begin{array}{l}4 \\
3\end{array}$ & $\begin{array}{l}2 \\
1\end{array}$ & 2 & 1 & $\begin{array}{l}15 \\
10\end{array}$ & $\begin{array}{l}3 \\
2\end{array}$ \\
\hline Chrysothamnus nauseosus & $\begin{array}{l}1979 \\
1980\end{array}$ & $\operatorname{tr}$ & & $\operatorname{tr}$ & 1 & 2 & 1 & $\begin{array}{l}8 \\
5\end{array}$ & $\begin{array}{l}2 \\
2\end{array}$ & $\begin{array}{l}2 \\
2\end{array}$ & $\begin{array}{l}1 \\
1\end{array}$ & $\begin{array}{l}\mathrm{tr} \\
\mathrm{tr}\end{array}$ & \\
\hline Chrysothamnus viscidiflorus & $\begin{array}{l}1979 \\
1980\end{array}$ & $\begin{array}{l}\operatorname{tr} \\
\operatorname{tr}\end{array}$ & & $\begin{array}{l}4 \\
1\end{array}$ & $\begin{array}{l}2 \\
0.4\end{array}$ & 1 & 0.3 & $\begin{array}{l}1 \\
1\end{array}$ & $\begin{array}{l}0.5 \\
0.3\end{array}$ & $\begin{array}{l}4 \\
2\end{array}$ & $\begin{array}{l}2 \\
1\end{array}$ & $\begin{array}{l}\operatorname{tr} \\
\operatorname{tr}\end{array}$ & \\
\hline Juniperus osteosperma & $\begin{array}{l}1979 \\
1980\end{array}$ & 1 & 1 & $\begin{array}{r}2 \\
26\end{array}$ & $\begin{array}{l}1 \\
4\end{array}$ & 28 & 6 & $\begin{array}{l}25 \\
27\end{array}$ & $\begin{array}{l}4 \\
6\end{array}$ & $\begin{array}{l}8 \\
5\end{array}$ & $\begin{array}{l}2 \\
2\end{array}$ & $\begin{array}{l}3 \\
4\end{array}$ & $\begin{array}{l}1 \\
2\end{array}$ \\
\hline Juniperus scopulorum & $\begin{array}{l}1979 \\
1980\end{array}$ & & & ${ }_{\operatorname{tr}}^{2}$ & 2 & $\operatorname{tr}$ & & $\begin{array}{l}\text { tr } \\
\text { tr }\end{array}$ & & tr & & $\operatorname{tr}$ & \\
\hline Pinus edulis & $\begin{array}{l}1979 \\
1980\end{array}$ & 2 & 2 & $\begin{array}{r}1 \\
24\end{array}$ & $\begin{array}{l}0.5 \\
4\end{array}$ & 21 & 4 & $\begin{array}{l}23 \\
19\end{array}$ & $\begin{array}{l}4 \\
4\end{array}$ & $\begin{array}{l}15 \\
14\end{array}$ & $\begin{array}{l}6 \\
5\end{array}$ & $\begin{array}{l}3 \\
1\end{array}$ & $\begin{array}{l}1 \\
0.3\end{array}$ \\
\hline Purshia tridentata & $\begin{array}{l}1979 \\
1980\end{array}$ & $\begin{array}{l}17 \\
19\end{array}$ & $\begin{array}{l}5 \\
2\end{array}$ & $\begin{array}{l}6 \\
1\end{array}$ & $\begin{array}{l}2 \\
1\end{array}$ & 2 & 1 & & & $\begin{array}{l}2 \\
1\end{array}$ & $\begin{array}{l}1 \\
1\end{array}$ & $\begin{array}{l}1 \\
2\end{array}$ & $\begin{array}{l}0.3 \\
1\end{array}$ \\
\hline Quercus gambelii & $\begin{array}{l}1979 \\
1980\end{array}$ & $\begin{array}{r}19 \\
8\end{array}$ & $\begin{array}{l}3 \\
2\end{array}$ & $\begin{array}{r}20 \\
4\end{array}$ & $\begin{array}{l}3 \\
1\end{array}$ & 5 & 2 & 1 & 1 & 2 & 1 & $\begin{array}{l}1 \\
1\end{array}$ & $\begin{array}{l}0.3 \\
0.3\end{array}$ \\
\hline Sarcobatus vermiculatus & $\begin{array}{l}1979 \\
1980\end{array}$ & & & & & & & $\begin{array}{r}\operatorname{tr} \\
3\end{array}$ & 1 & $\begin{array}{l}\operatorname{tr} \\
14\end{array}$ & 6 & & \\
\hline Symphoricarpos oreophilus & $\begin{array}{l}1979 \\
1980\end{array}$ & $\underset{\operatorname{tr}}{1}$ & 1 & $\begin{array}{c}1 \\
\operatorname{tr}\end{array}$ & 0.4 & 6 & 2 & $\begin{array}{l}2 \\
2\end{array}$ & $\begin{array}{l}1 \\
1\end{array}$ & $\begin{array}{l}3 \\
2\end{array}$ & $\begin{array}{l}1 \\
1\end{array}$ & $\mathrm{tr}_{2}$ & 1 \\
\hline Tetradymia canescens & $\begin{array}{l}1979 \\
1980\end{array}$ & $\begin{array}{l}\mathrm{tr} \\
\mathrm{tr}\end{array}$ & & tr & & $\operatorname{tr}$ & & $\begin{array}{l}1 \\
3\end{array}$ & $\begin{array}{l}1 \\
1\end{array}$ & $\begin{array}{l}4 \\
6\end{array}$ & $\begin{array}{l}1 \\
2\end{array}$ & $\operatorname{tr}$ & \\
\hline All trees and shrubs & $\begin{array}{l}1979 \\
1980\end{array}$ & $\begin{array}{l}93 \\
88\end{array}$ & $\begin{array}{l}1 \\
3\end{array}$ & $\begin{array}{l}96 \\
99\end{array}$ & $\begin{array}{l}1 \\
1\end{array}$ & 90 & 3 & $\begin{array}{l}82 \\
81\end{array}$ & $\begin{array}{l}3 \\
3\end{array}$ & $\begin{array}{l}66 \\
75\end{array}$ & $\begin{array}{l}5 \\
4\end{array}$ & $\begin{array}{l}41 \\
46\end{array}$ & $\begin{array}{l}2 \\
7\end{array}$ \\
\hline $\begin{array}{l}\text { Forbs } \\
\quad \text { Artemisia frigida }\end{array}$ & $\begin{array}{l}1979 \\
1980\end{array}$ & $\operatorname{tr}$ & & $\begin{array}{l}\mathrm{tr} \\
\mathrm{tr}\end{array}$ & & $\operatorname{tr}$ & & $\begin{array}{l}3 \\
3\end{array}$ & $\begin{array}{l}1 \\
1\end{array}$ & $\begin{array}{l}3 \\
2\end{array}$ & $\begin{array}{l}1 \\
0.5\end{array}$ & $\begin{array}{l}\operatorname{tr} \\
\operatorname{tr}\end{array}$ & \\
\hline Artemisia ludoviciana & $\begin{array}{l}1979 \\
1980\end{array}$ & $\begin{array}{l}\mathrm{tr} \\
\mathrm{tr}\end{array}$ & & $\begin{array}{l}\mathrm{tr} \\
\mathrm{tr}\end{array}$ & & $\operatorname{tr}$ & & $\begin{array}{l}2 \\
2\end{array}$ & $\begin{array}{l}1 \\
1\end{array}$ & $\begin{array}{l}8 \\
9\end{array}$ & $\begin{array}{l}2 \\
1\end{array}$ & $\begin{array}{l}1 \\
\operatorname{tr}\end{array}$ & 0.4 \\
\hline Comandra umbellata & $\begin{array}{l}1979 \\
1980\end{array}$ & ${ }_{5}^{\operatorname{tr}}$ & 2 & & & & & & & & & $\operatorname{tr}$ & \\
\hline Cryptantha sericea & $\begin{array}{l}1979 \\
1980\end{array}$ & $\operatorname{tr}$ & & tr & & $\operatorname{tr}$ & & $\begin{array}{l}\text { tr } \\
\text { tr }\end{array}$ & & $\mathrm{tr}_{1}$ & 0.3 & $\begin{array}{l}5 \\
4\end{array}$ & $\begin{array}{l}1 \\
0.5\end{array}$ \\
\hline Eriogonum lonchophyllum & $\begin{array}{l}1979 \\
1980\end{array}$ & $\underset{\operatorname{tr}}{1}$ & 0.4 & & & $\operatorname{tr}$ & & $\begin{array}{l}8 \\
7\end{array}$ & $\begin{array}{l}2 \\
1\end{array}$ & $\begin{array}{l}9 \\
9\end{array}$ & $\begin{array}{l}3 \\
3\end{array}$ & $\operatorname{tr}$ & \\
\hline Eriogonum umbellatum & $\begin{array}{l}1979 \\
1980\end{array}$ & $\underset{\operatorname{tr}}{1}$ & 0.5 & & & & & & & & & $\begin{array}{l}1 \\
2\end{array}$ & $\begin{array}{l}0.4 \\
1\end{array}$ \\
\hline Haplopappus nuttallii & $\begin{array}{l}1979 \\
1980\end{array}$ & $\begin{array}{l}1 \\
2\end{array}$ & $\begin{array}{l}0.4 \\
1\end{array}$ & $\operatorname{tr}$ & & $\operatorname{tr}$ & & $\begin{array}{r}\mathbf{t r} \\
1\end{array}$ & 0.3 & $\operatorname{tr}^{2}$ & 1 & & \\
\hline Hymenopappus filifolius & $\begin{array}{l}1979 \\
1980\end{array}$ & & & & & & & & & 2 & 1 & $\begin{array}{l}\text { tr } \\
\text { tr }\end{array}$ & \\
\hline Lupinus caudatus & $\begin{array}{l}1979 \\
1980\end{array}$ & 1 & 1 & $\operatorname{tr}^{2}$ & 1 & 2 & 1 & $\begin{array}{l}\operatorname{tr} \\
\mathrm{tr}\end{array}$ & & $\operatorname{tr}$ & & $\begin{array}{l}1 \\
3\end{array}$ & $\begin{array}{l}0.2 \\
1\end{array}$ \\
\hline Mertensia lanceolata & $\begin{array}{l}1979 \\
1980\end{array}$ & $\begin{array}{l}\mathrm{tr} \\
\mathrm{tr}\end{array}$ & & $\operatorname{tr}$ & & tr & & & & & & $\begin{array}{l}2 \\
4\end{array}$ & $\begin{array}{l}1 \\
1\end{array}$ \\
\hline Phlox hoodii & $\begin{array}{l}1979 \\
1980\end{array}$ & & & & & tr & & 1 & 1 & $\begin{array}{l}1 \\
1\end{array}$ & $\begin{array}{l}1 \\
0.3\end{array}$ & $\begin{array}{l}3 \\
3\end{array}$ & $\begin{array}{l}1 \\
1\end{array}$ \\
\hline Senecio mutabilis & $\begin{array}{l}1979 \\
1980\end{array}$ & $\begin{array}{l}\mathrm{tr} \\
\mathrm{tr}\end{array}$ & & $\operatorname{tr}$ & & & & & & & & $\begin{array}{l}2 \\
1\end{array}$ & $\begin{array}{l}0.4 \\
0.2\end{array}$ \\
\hline All forbs & $\begin{array}{l}1979 \\
1980\end{array}$ & $\begin{array}{r}6 \\
12\end{array}$ & $\begin{array}{l}1 \\
3\end{array}$ & $\begin{array}{l}4 \\
1\end{array}$ & $\begin{array}{l}1 \\
0.5\end{array}$ & 4 & 1 & $\begin{array}{l}15 \\
17\end{array}$ & $\begin{array}{l}2 \\
3\end{array}$ & $\begin{array}{l}29 \\
24\end{array}$ & $\begin{array}{l}4 \\
4\end{array}$ & $\begin{array}{l}18 \\
21\end{array}$ & $\begin{array}{l}2 \\
3\end{array}$ \\
\hline
\end{tabular}


Table 1. continued.

\begin{tabular}{|c|c|c|c|c|c|c|c|c|c|c|c|c|c|}
\hline \multirow[b]{2}{*}{ Species } & \multirow[b]{2}{*}{ Year } & \multicolumn{2}{|c|}{ October } & \multicolumn{2}{|c|}{ November } & \multicolumn{2}{|c|}{ January } & \multicolumn{2}{|c|}{ February } & \multicolumn{2}{|c|}{ March } & \multicolumn{2}{|c|}{ April } \\
\hline & & $\overline{\bar{x}}$ & $\mathrm{SE}$ & $\bar{x}$ & $\mathrm{SE}$ & $\bar{x}$ & $\mathrm{SE}$ & $\bar{x}$ & $\overline{\mathrm{SE}}$ & $\bar{x}$ & SE & $\bar{x}$ & $\mathrm{SE}$ \\
\hline \multicolumn{14}{|l|}{ Grasses } \\
\hline \multirow[t]{2}{*}{ Agropyron spp. ${ }^{2}$} & 1979 & & & & & & & $\operatorname{tr}$ & & $\operatorname{tr}$ & & 3 & 1 \\
\hline & 1980 & $\operatorname{tr}$ & & $\operatorname{tr}$ & & 1 & 0.3 & $\operatorname{tr}$ & & $\operatorname{tr}$ & & 2 & 1 \\
\hline \multirow[t]{2}{*}{ Carex spp. ${ }^{3}$} & 1979 & $\operatorname{tr}$ & & & & & & tr & & 1 & 0.3 & 9 & 2 \\
\hline & 1980 & $\operatorname{tr}$ & & & & tr & & 1 & 0.2 & $\mathrm{tr}$ & & 8 & 2 \\
\hline \multirow[t]{2}{*}{ Poa spp. ${ }^{4}$} & 1979 & $\operatorname{tr}$ & & $\mathrm{tr}$ & & & & $\operatorname{tr}$ & & 2 & 1 & 23 & 2 \\
\hline & 1980 & $\operatorname{tr}$ & & $\operatorname{tr}$ & & 2 & 1 & 1 & 0.4 & $\operatorname{tr}$ & & 23 & 5 \\
\hline \multirow[t]{2}{*}{ All grasses } & 1979 & $\operatorname{tr}$ & & $\mathrm{tr}$ & & & & 3 & 2 & 5 & 1 & 41 & 3 \\
\hline & 1980 & $\operatorname{tr}$ & & 1 & 1 & 6 & 1 & 2 & 1 & 1 & 0.3 & 34 & 4 \\
\hline
\end{tabular}

'Percentages are means of 8 individual deer diets except in April 1979-80 when there were 6 deer.

${ }^{2}$ Includes Agropyron spicatum, A. smithii, A. cristatum, and possibly others.

Includes Carex geophila, C. pityophila, and possibly others.

"Includes Poa fendleriana, $P$. pratensis, $P$. sandbergii, and possibly others.

num lonchophyllum), mat penstemon (Penstemon caespitosus), lupine, and sulphur eriogonum retained some color or succulence and were selected over species already dry. Grasses were essentially ignored during October.

By late November, forbs and grasses were covered by snow and largely unavailable on ridge tops and north aspects used by wild deer. Shrubs and trees dominating diets that month included serviceberry, mountain mahogany, juniper, pinyon, bitterbrush, and oak. January foraging sites (Year 2 only) included south exposures with shallower snow and some bare ground. As a result, forbs and grasses were more available than on sites in November and diet percentages of both increased, especially on Area 2. Leaves of serviceberry, mahogany and shadscale (Atriplex confertifolia) which accumulated under shrubs were readily consumed during both January and February. Sites for February and March trials were on south aspects and use of herbs, particularly forbs, continued to increase with greatest consumption again on Area 2. In April, new growth of many species was available but grass, mostly bluegrasses, dominated all diets. Four to 5 times more grass than browse was eaten on Area 2 while proportions of both were similar on Area 1.

Diet proportions of browse, forbs, and grasses differed $(P<0.05)$ more between areas than yea rs (Table 3 ). Higher browse consumption $(P<0.05)$ on Area 1 than Area 2 most months was usually compensated by lower grass use. Use of some of the more common species was unrelated to their availability. Pinyon and juniper were abundant on all areas during all sampling periods, but deer made greatest use of them from November to March when snow was present. During November of Year 2, these 2 species increased sharply in diets on both areas over the same month in Year 1. No specific reason can be offered but, in Year 2, snow was deeper $(-40$ $v s-25 \mathrm{~cm}$ ) and a plentiful crop of juniper berries was attractive to the deer.

In contrast, snowberry and sagebrush were abundant species eaten in relatively small amounts. Snowberry contributed $\leq 3 \%$ to diets both years. The most sagebrush in a composite diet was $11 \%$ and the highest for an individual deer was 19\%. Previously in Piceance Basin, Carhart (1943) reported 37 and 27\% sagebrush in rumen samples during winter and spring, respectively. In a review of mule deer food habits literature, Kufeld et al. (1973) reported sagebrush use as generally "heavy" during winter and spring. However, comparing results obtained with different methods can be misleading. For example, feeding site analysis and long-range observation of animals feeding tend to overestimate percent shrubs in a diet (Wallmo et al. 1973). Rumen analyses produce varied results depending on the kinds and amounts of forages consumed (Norris 1943, Bergerud and Russell 1964, Gaare et al. 1977).
A potential problem with consumption of sagebrush, and also juniper and pinyon, is inhibition of rumen microbial function by volatile oils. This effect has been predicted with both sagebrush and juniper at dietary levels of $15-30 \%$ (Nagy et al. 1964, Jobman 1972). However, Cluff et al. (1982) concluded most monoterpenoids in sagebrush are lost early in the digestive process and their inhibitory effect on microbial activity is probably less than originally conceived. Relevance of this question is highlighted in this study during Year 2 when combined proportions of juniper, pinyon, and sagebrush, all fresh material, averaged $45 \%$ of November to March diets. This high level of use was assumed to continue while deer were in the holding pasture between sampling periods but they exhibited no visible ill effects other than the weight loss expected over winter.

Serviceberry, mahogany, and bitterbrush were ubiquitous species often taken in substantial quantities. A low proportion or absence of any one of them in a diet usually reflected low availability on the specific sites grazed by the deer. Oak, although restricted in distribution at lower elevations on Area 1 and scarce on Area 2 , was also eaten whenever encountered. A variety of other species, primarily forbs, were also frequently eaten whenever found. However, low abundance or availability during winter precluded the possibility of many of these species to be major diet items.

\section{Diet Quality}

Winter-long trends in $\mathrm{CP}$ content of deer diets were similar on both areas each year (Fig. 1). October dietary CP, 8-10\%, dropped to $5-7 \%$ from November through March and then increased ito 13-23\% in April. A similar pattern was displayed with dietary IVDDM except early in Year 1 when there was no distinct drop in IVDDM from October to November on either area. October to March IVDDM ranged from $24-37 \%$ and increased to $43-62 \%$ in April. These levels of CP and IVDDM were quite close to values reported by Hobbs et al. (1983) for mule deer on a montane winter range in Colorado.

From October-March, CP content of both grasses and forbs in deer diets averaged nearly 2 percentage points lower than for browse. During the same period, IVDDM of grasses and forbs averaged about 3 and 8 percentage points, respectively, lower than browse. Thus, browse was primarily responsible for maintaining winter diet quality. In April, the sharp increased in dietary $\mathrm{CP}$ and IVDDM reflected a shift in feeding emphasis to new growth of grasses and forbs. Dietary CP and IVDDM were both higher $(P<0.05)$ in April each year on Area 2 where forbs and grasses composed $>80 \%$ of diets compared to $<60 \%$ on Area 1 .

Grass species were major items in 7 of 10 January-March diets. The IVDDM content of grasses ranged from $21-35 \%$ which was similar to that for most browse species in those same diets. This 
Table 2. Percentages of major forage species in tame mule deer diets on pinyon-juniper-sagebrush winter range in Piceance Basin, Colorado, 1978-79 and 1979-80.1

\begin{tabular}{|c|c|c|c|c|c|c|c|c|c|c|c|c|c|}
\hline \multirow[b]{2}{*}{ Species } & \multirow[b]{2}{*}{ Year } & \multicolumn{2}{|c|}{ October } & \multicolumn{2}{|c|}{ November } & \multicolumn{2}{|c|}{ January } & \multicolumn{2}{|c|}{ February } & \multicolumn{2}{|c|}{ March } & \multicolumn{2}{|c|}{ April } \\
\hline & & $\overline{\vec{x}}$ & $\overline{\mathrm{SE}}$ & $\bar{x}$ & $\overline{S E}$ & $\bar{x}$ & SE & $\bar{x}$ & SE & $\bar{x}$ & $\overline{\mathrm{SE}}$ & $\bar{x}$ & SE \\
\hline $\begin{array}{l}\text { Trees and shrubs } \\
\text { Amelanchier utahensis }\end{array}$ & $\begin{array}{l}1979 \\
1980\end{array}$ & $\begin{array}{l}11 \\
25\end{array}$ & $\begin{array}{l}2 \\
4\end{array}$ & 3 & 1 & $\operatorname{tr}$ & & $\begin{array}{l}\operatorname{tr} \\
\operatorname{tr}\end{array}$ & & $\operatorname{tr}$ & & $\begin{array}{l}3 \\
2\end{array}$ & $\begin{array}{l}1 \\
1\end{array}$ \\
\hline Artemisia tridentata & $\begin{array}{l}1979 \\
1980\end{array}$ & tr & & $\begin{array}{l}4 \\
3\end{array}$ & $\begin{array}{l}1 \\
1\end{array}$ & 1 & 0.2 & $\begin{array}{l}5 \\
7\end{array}$ & $\begin{array}{l}1 \\
2\end{array}$ & $\begin{array}{l}4 \\
3\end{array}$ & $\begin{array}{l}1 \\
1\end{array}$ & $\begin{array}{l}4 \\
1\end{array}$ & $\begin{array}{l}2 \\
0.4\end{array}$ \\
\hline Cercocarpus montanus & $\begin{array}{l}1979 \\
1980\end{array}$ & $\begin{array}{r}2 \\
22\end{array}$ & $\begin{array}{l}1 \\
5\end{array}$ & $\begin{array}{l}47 \\
18\end{array}$ & $\begin{array}{l}5 \\
5\end{array}$ & 4 & 1 & $\begin{array}{l}\mathrm{tr} \\
\mathrm{tr}\end{array}$ & & $\begin{array}{l}3 \\
3\end{array}$ & $\begin{array}{l}1 \\
1\end{array}$ & ${ }_{\operatorname{tr}}^{2}$ & 1 \\
\hline Chrysothamnus nauseosus & $\begin{array}{l}1979 \\
1980\end{array}$ & & & $\operatorname{tr}$ & & 1 & 0.4 & $\begin{array}{r}17 \\
2\end{array}$ & $\begin{array}{l}5 \\
1\end{array}$ & $\begin{array}{l}5 \\
2\end{array}$ & $\begin{array}{l}1 \\
1\end{array}$ & $\mathrm{tr}$ & \\
\hline Chrysothamnus viscidiflorus & $\begin{array}{l}1979 \\
1980\end{array}$ & $\begin{array}{l}\mathbf{t r} \\
\mathbf{t r}\end{array}$ & & $\begin{array}{l}\operatorname{tr} \\
\operatorname{tr}\end{array}$ & & $\operatorname{tr}$ & & $\mathrm{tr}_{3}$ & 1 & $\begin{array}{l}\operatorname{tr} \\
\operatorname{tr}\end{array}$ & & $\mathrm{tr}$ & \\
\hline Juniperus osteosperma & $\begin{array}{l}1979 \\
1980\end{array}$ & $\mathbf{t r}_{1}$ & 1 & $\begin{array}{l}10 \\
54\end{array}$ & $\begin{array}{l}4 \\
7\end{array}$ & 26 & 5 & $\begin{array}{l}17 \\
22\end{array}$ & $\begin{array}{l}3 \\
5\end{array}$ & $\begin{array}{r}9 \\
15\end{array}$ & $\begin{array}{l}2 \\
4\end{array}$ & 1 & 0.2 \\
\hline Leptodactylon pungens & $\begin{array}{l}1979 \\
1980\end{array}$ & tr & & & & $\operatorname{tr}$ & & $\operatorname{tr}^{2}$ & 1 & $\mathrm{tr}_{1}$ & 0.3 & & \\
\hline Pinus edulis & $\begin{array}{l}1979 \\
1980\end{array}$ & tr & & $\begin{array}{r}3 \\
15\end{array}$ & $\begin{array}{l}1 \\
3\end{array}$ & 26 & 4 & $\begin{array}{l}8 \\
2\end{array}$ & $\begin{array}{l}4 \\
1\end{array}$ & $\begin{array}{l}1 \\
5\end{array}$ & $\begin{array}{l}1 \\
3\end{array}$ & $\operatorname{tr}$ & \\
\hline Purshia tridentata & $\begin{array}{l}1979 \\
1980\end{array}$ & $\begin{array}{l}62 \\
36\end{array}$ & $\begin{array}{l}7 \\
4\end{array}$ & $\begin{array}{r}26 \\
7\end{array}$ & $\begin{array}{l}4 \\
1\end{array}$ & 12 & 3 & $\begin{array}{l}1 \\
5\end{array}$ & $\begin{array}{l}0.3 \\
2\end{array}$ & $\begin{array}{r}11 \\
4\end{array}$ & $\begin{array}{l}3 \\
1\end{array}$ & $\begin{array}{l}7 \\
9\end{array}$ & $\begin{array}{l}1 \\
1\end{array}$ \\
\hline Sarcobatus vermiculatus & $\begin{array}{l}1979 \\
1980\end{array}$ & & & $\operatorname{tr}$ & & $\operatorname{tr}$ & & 12 & 3 & 2 & 1 & & \\
\hline Symphoricarpos oreophilus & $\begin{array}{l}1979 \\
1980\end{array}$ & ${ }_{\mathrm{tr}}^{2}$ & 1 & $\operatorname{tr}$ & & tr & & $\begin{array}{l}\text { tr } \\
\text { tr }\end{array}$ & & $\begin{array}{l}\text { tr } \\
\text { tr }\end{array}$ & & $\mathbf{t r}$ & \\
\hline Tetradymia canescens & $\begin{array}{l}1979 \\
1980\end{array}$ & $\begin{array}{l}\text { tr } \\
\text { tr }\end{array}$ & & 2 & 1 & $\operatorname{tr}$ & & $\mathrm{tr}$ & 0.4 & $\begin{array}{l}4 \\
2\end{array}$ & $\begin{array}{l}1 \\
1\end{array}$ & & \\
\hline Xanthocephalum sarothrae & $\begin{array}{l}1979 \\
1980\end{array}$ & tr & & $\operatorname{tr}$ & & tr & & $\begin{array}{l}\text { tr } \\
\text { tr }\end{array}$ & & $\begin{array}{l}4 \\
4\end{array}$ & $\begin{array}{l}1 \\
1\end{array}$ & & \\
\hline All trees and shrubs & $\begin{array}{l}1979 \\
1980\end{array}$ & $\begin{array}{l}79 \\
85\end{array}$ & $\begin{array}{l}5 \\
3\end{array}$ & $\begin{array}{l}95 \\
98\end{array}$ & $\begin{array}{l}2 \\
1\end{array}$ & 70 & 6 & $\begin{array}{l}64 \\
44\end{array}$ & $\begin{array}{l}3 \\
4\end{array}$ & $\begin{array}{l}44 \\
42\end{array}$ & $\begin{array}{l}3 \\
3\end{array}$ & $\begin{array}{l}18 \\
13\end{array}$ & $\begin{array}{l}3 \\
1\end{array}$ \\
\hline Forbs & & & & & & & & & & & & & \\
\hline Agoseris aurantiaca & $\begin{array}{l}1979 \\
1980\end{array}$ & & & & & & & & & & & $\begin{array}{l}2 \\
2\end{array}$ & $\begin{array}{l}0.4 \\
0.3\end{array}$ \\
\hline Comandra umbellata & $\begin{array}{l}1979 \\
1980\end{array}$ & $\begin{array}{l}3 \\
8\end{array}$ & $\begin{array}{l}1 \\
2\end{array}$ & & & & & & & & & $\operatorname{tr}$ & \\
\hline Cryptantha sericea & $\begin{array}{l}1979 \\
1980\end{array}$ & $\begin{array}{l}\text { tr } \\
\text { tr }\end{array}$ & & & & tr & & $\begin{array}{r}\operatorname{tr} \\
2\end{array}$ & 1 & $\begin{array}{l}1 \\
1\end{array}$ & $\begin{array}{l}0.3 \\
0.1\end{array}$ & $\begin{array}{l}1 \\
1\end{array}$ & $\begin{array}{l}0.3 \\
0.2\end{array}$ \\
\hline Eriogonum lonchophyllum & $\begin{array}{l}1979 \\
1980\end{array}$ & $\operatorname{tr}^{5}$ & 2 & & & 2 & 1 & $\begin{array}{r}3 \\
12\end{array}$ & $\begin{array}{l}1 \\
2\end{array}$ & $\begin{array}{r}5 \\
10\end{array}$ & $\begin{array}{l}1 \\
1\end{array}$ & & \\
\hline Eriogonum umbellatum & $\begin{array}{l}1979 \\
1980\end{array}$ & $\begin{array}{l}3 \\
1\end{array}$ & $\begin{array}{l}1 \\
0.1\end{array}$ & & & & & tr & & & & tr & \\
\hline Haplopappus nuttallii & $\begin{array}{l}1979 \\
1980\end{array}$ & $\begin{array}{l}\mathrm{tr} \\
\mathrm{tr}\end{array}$ & & $\operatorname{tr}$ & & 1 & 1 & $\begin{array}{l}1 \\
9\end{array}$ & $\begin{array}{l}0.4 \\
1\end{array}$ & $\begin{array}{r}10 \\
4\end{array}$ & $\begin{array}{l}3 \\
1\end{array}$ & & \\
\hline Hymenopappus filifolius & $\begin{array}{l}1979 \\
1980\end{array}$ & & & & & 3 & 1 & $\begin{array}{l}7 \\
4\end{array}$ & $\begin{array}{l}2 \\
1\end{array}$ & $\begin{array}{r}15 \\
6\end{array}$ & $\begin{array}{l}5 \\
1\end{array}$ & $\operatorname{tr}$ & \\
\hline Lupinus caudatus & $\begin{array}{l}1979 \\
1980\end{array}$ & $\begin{array}{l}3 \\
1\end{array}$ & $\begin{array}{l}1 \\
0.3\end{array}$ & $\operatorname{tr}^{1}$ & 0.2 & & & $\operatorname{tr}$ & & $\operatorname{tr}$ & & 1 & 0.2 \\
\hline Penstemon caespitosus & $\begin{array}{l}1979 \\
1980\end{array}$ & $\operatorname{tr}^{2}$ & 1 & & & & & & & & & & \\
\hline Penstemon osterhoutii & $\begin{array}{l}1979 \\
1980\end{array}$ & & & & & 2 & 1 & $\begin{array}{c}\operatorname{tr} \\
8\end{array}$ & 4 & $\begin{array}{r}4 \\
10\end{array}$ & $\begin{array}{l}1 \\
3\end{array}$ & & \\
\hline Penstemon watsonii & $\begin{array}{l}1979 \\
1980\end{array}$ & $\begin{array}{l}1 \\
2\end{array}$ & $\begin{array}{l}0.4 \\
1\end{array}$ & & & & & & & & & & \\
\hline Phlox hoodii & $\begin{array}{l}1979 \\
1980\end{array}$ & & & $\operatorname{tr}$ & & $\operatorname{tr}$ & & $\operatorname{tr}_{1}$ & 1 & $\begin{array}{l}2 \\
4\end{array}$ & $\begin{array}{l}1 \\
1\end{array}$ & $\begin{array}{l}1 \\
1\end{array}$ & $\begin{array}{l}1 \\
0.2\end{array}$ \\
\hline Senecio mutabilis & $\begin{array}{l}1979 \\
1980\end{array}$ & $\begin{array}{l}\mathrm{tr} \\
\mathrm{tr}\end{array}$ & & & & & & & & & & $\begin{array}{l}2 \\
1\end{array}$ & $\begin{array}{l}1 \\
0.2\end{array}$ \\
\hline Zygadenus venosus & $\begin{array}{l}1979 \\
1980\end{array}$ & & & & & & & & & & & $\begin{array}{l}3 \\
2\end{array}$ & $\begin{array}{l}1 \\
1\end{array}$ \\
\hline
\end{tabular}


Table 2. Continued.

\begin{tabular}{|c|c|c|c|c|c|c|c|c|c|c|c|c|c|}
\hline \multirow[b]{2}{*}{ Species } & \multirow[b]{2}{*}{ Year } & \multicolumn{2}{|c|}{ October } & \multicolumn{2}{|c|}{ November } & \multicolumn{2}{|c|}{ January } & \multicolumn{2}{|c|}{ February } & \multicolumn{2}{|c|}{ March } & \multicolumn{2}{|c|}{ April } \\
\hline & & $\bar{x}$ & SE & $\bar{x}$ & SE & $\overline{\boldsymbol{x}}$ & SE & $\bar{x}$ & SF & $\bar{x}$ & SE & $\bar{x}$ & SE \\
\hline All forbs & $\begin{array}{l}1979 \\
1980\end{array}$ & $\begin{array}{l}20 \\
15\end{array}$ & $\begin{array}{l}5 \\
3\end{array}$ & $\begin{array}{l}2 \\
1\end{array}$ & $\begin{array}{l}1 \\
0.4\end{array}$ & 10 & 3 & $\begin{array}{l}16 \\
43\end{array}$ & $\begin{array}{l}3 \\
6\end{array}$ & $\begin{array}{l}44 \\
38\end{array}$ & $\begin{array}{l}5 \\
3\end{array}$ & $\begin{array}{r}12 \\
9\end{array}$ & $\begin{array}{l}2 \\
1\end{array}$ \\
\hline $\begin{array}{l}\text { Grasses } \\
\quad \text { Agropyron spp. }{ }^{2}\end{array}$ & $\begin{array}{l}1979 \\
1980\end{array}$ & $\begin{array}{l}\mathrm{tr} \\
\mathrm{tr}\end{array}$ & & $\begin{array}{l}\mathrm{tr} \\
\mathrm{tr}\end{array}$ & & 5 & 2 & $\begin{array}{l}4 \\
1\end{array}$ & $\begin{array}{l}2 \\
1\end{array}$ & $\begin{array}{l}\mathrm{tr} \\
\mathrm{tr}\end{array}$ & & $\begin{array}{l}3 \\
4\end{array}$ & $\begin{array}{l}0.4 \\
1\end{array}$ \\
\hline Carex spp. ${ }^{3}$ & $\begin{array}{l}1979 \\
1980\end{array}$ & $\begin{array}{l}\text { tr } \\
\text { tr }\end{array}$ & & $\mathbf{t r}$ & & 1 & 0.2 & $\mathbf{t r}_{1}$ & 0.3 & $\begin{array}{l}2 \\
8\end{array}$ & $\begin{array}{l}1 \\
2\end{array}$ & $\operatorname{tr}$ & \\
\hline Koeleria cristata & $\begin{array}{l}1979 \\
1980\end{array}$ & $\begin{array}{l}\operatorname{tr} \\
\operatorname{tr}\end{array}$ & & $\operatorname{tr}$ & & $\operatorname{tr}$ & & tr & & $\operatorname{tr}^{2}$ & 1 & 5 & 1 \\
\hline Oryzopsis hymenoides & $\begin{array}{l}1979 \\
1980\end{array}$ & $\begin{array}{l}\mathrm{tr} \\
\mathrm{tr}\end{array}$ & & $\mathrm{tr}_{1}$ & 0.5 & 6 & 1 & $\begin{array}{l}7 \\
4\end{array}$ & $\begin{array}{l}2 \\
1\end{array}$ & $\begin{array}{l}1 \\
7\end{array}$ & $\begin{array}{l}0.4 \\
2\end{array}$ & $\operatorname{tr}$ & \\
\hline Poa spp. ${ }^{4}$ & $\begin{array}{l}1979 \\
1980\end{array}$ & $\begin{array}{l}\operatorname{tr} \\
\operatorname{tr}\end{array}$ & $\mathbf{t r}$ & 1 & 0.5 & 1 & 0.3 & $\underset{3}{\operatorname{tr}}$ & 1 & $\begin{array}{l}1 \\
3\end{array}$ & $\begin{array}{l}0.4 \\
1\end{array}$ & $\begin{array}{l}54 \\
72\end{array}$ & $\begin{array}{l}3 \\
2\end{array}$ \\
\hline Stipa comata & $\begin{array}{l}1979 \\
1980\end{array}$ & $\begin{array}{l}\mathrm{tr} \\
\mathrm{tr}\end{array}$ & & $\operatorname{tr}$ & 0.2 & 3 & 1 & $\begin{array}{l}7 \\
1\end{array}$ & $\begin{array}{l}2 \\
1\end{array}$ & $\operatorname{tr}^{3}$ & 1 & & \\
\hline All grasses & $\begin{array}{l}1979 \\
1980\end{array}$ & $\operatorname{tr}^{1}$ & 1 & $\begin{array}{l}3 \\
1\end{array}$ & $\begin{array}{l}1 \\
0.3\end{array}$ & 20 & 3 & $\begin{array}{l}21 \\
13\end{array}$ & $\begin{array}{l}5 \\
3\end{array}$ & $\begin{array}{l}12 \\
21\end{array}$ & $\begin{array}{l}2 \\
4\end{array}$ & $\begin{array}{l}70 \\
77\end{array}$ & $\begin{array}{l}4 \\
2\end{array}$ \\
\hline
\end{tabular}

'Percentages are means of 8 individual deer diets except in April 1979-80 when there were 6 deer.

IIncludes Agropyron spicatum, $A$. smithii, A. cristatum, and possibly others.

${ }^{3}$ Includes Carex geophila, C. pityophila, and possibly others.

${ }^{4}$ Includes Poa fendleriana, $P$. pratensis, $P$. sandbergii, and possibly others.

contrasts with the higher IVDDM content of grass over browse in elk winter diets (Hobbs et al. 1981) and in the standing crop of forage (Cook and Harris 1968, Wallmo et al. 1977). A repeat of IVDDM analyses on Year I forage samples supported the relationship between grass and browse IVDDM in this study as did IVDDM analyses of the standing crop of winter forage in another study in Piceance Basin during the 1980 winter (L. Carpenter, unpubl. data).

Significant differences $(P<0.05)$ in dietary CP and IVDDM were more common between areas than between years (Table 3). However, significance was due mostly to low variability among animals and smail differences in mean values are probably offset by variability in forage collection and analysis procedures.

\section{Discussion}

Availability, as well as palatability, was responsible for some spatial and temporal differences in diet compositions of tame deer. This was indicated, in part, by the shift from nearly $100 \%$ browse diets in early winter to more forbs and grasses as snow decreased and deer moved to south a spects. In spite of large differences in diet compositions among animals and sampling periods, deer selected forage mixes to help maintain a fairly consistent diet quality through the critical mid-to late winter period. This feeding strategy is similar to that observed in elk during winter in Colorado (Hobbs et al. 1981).

Estimates of minimum CP requirements for maintenance of deer generally vary between 5-7\% (Einarson 1946, Bissell and Strong 1955, Robbins et al. 1975, Holter et al. 1979). November to March dietary CP levels were usually within this range. Diet IVDDM, on the other hand, was much less than the approximate $50 \%$ digestibility level considered by Ammann et al. (1973) as maintenance for a ruminant. However, there are several possibilities for increased diet levels of both CP and IVDDM above those estimated. One is consumption of greater proportions of more nutritious forage such as sagebrush. However, nutritional value of sagebrush in vivo has yet to be substantiated. Another is that animals may select more nutritious forage than is accomplished by hand-picking (Weir and Torrell 1959). A third possibility is through associative effects during the digestive process (Cook and Harris 1967, Milchunas et al. 1978). Finally, soil contamination resulted in high ash values for some species, particularly several low growing forbs, causing low $\mathrm{CP}$ and digestibilities on a dry matter basis. On the negative side, there may be a greater IVDDM deficit than indicated as loss of voltile oils by oven-drying and grinding forage samples may produce inflated IVDDM values for pinyon, juniper, and sagebrush.

The role of various forages in deer nutrition is poorly under-

Table 3. Results of paired and unpaired $t$ tests of attributes of tame mule deer diets on pinyon-juniper-mixed shrub (Area 1 ) and pinyon-juniper-sagebrush (Area 2) winter range in Piceance Basin, Colorado, during October-April, 1978-79 and 1979-80.,2

\begin{tabular}{|c|c|c|c|c|c|c|c|c|c|c|c|c|c|c|c|c|c|c|c|c|c|}
\hline \multirow[b]{3}{*}{ Diet attribute } & \multicolumn{11}{|c|}{ Area 1 vs Area 2} & \multicolumn{10}{|c|}{$1978-79$ vs $1979-80$} \\
\hline & \multicolumn{5}{|c|}{$1978-79$} & \multicolumn{5}{|c|}{$1979-80$} & \multirow[b]{2}{*}{$\mathbf{A}$} & \multicolumn{4}{|c|}{ Area 1} & \multirow[b]{2}{*}{$\mathbf{A}$} & \multicolumn{4}{|c|}{ Area 2} & \multirow[b]{2}{*}{$\mathbf{A}$} \\
\hline & O & $\mathbf{N}$ & $F$ & $\mathbf{M}$ & A & 0 & $\mathbf{N}$ & $\mathbf{J}$ & $\mathbf{F}$ & $\mathbf{M}$ & & $\mathbf{O}$ & $\mathbf{N}$ & $\mathbf{F}$ & $\mathbf{M}$ & & $\mathbf{O}$ & $\mathbf{N}$ & $\mathbf{F}$ & $\mathbf{M}$ & \\
\hline Trees and shrubs & $\mathbf{H}^{3}$ & - & $\mathbf{H}$ & $\mathrm{H}$ & $\mathbf{H}$ & - & - & $\mathbf{H}$ & $\mathbf{H}$ & $\mathbf{H}$ & $\mathbf{H}$ & - & - & - & $\mathbf{L}$ & - & - & - & $\mathbf{H}$ & - & - \\
\hline Forbs & L & $\mathbf{H}$ & - & $\mathbf{L}$ & $\mathbf{H}$ & - & - & L & $\mathbf{L}$ & $\mathrm{L}$ & $\mathbf{H}$ & - & - & - & - & - & - & - & $\mathbf{L}$ & - & - \\
\hline Grasses & - & - & $\mathbf{L}$ & $\mathrm{L}$ & $\mathrm{L}$ & - & - & L & $\mathrm{L}$ & $\mathrm{L}$ & $\mathbf{L}$ & 一 & - & - & $\mathbf{H}$ & - & - & - & - & - & - \\
\hline Crude protein & 一 & $\mathbf{L}$ & - & $\mathrm{H}$ & $\mathbf{L}$ & $\mathbf{H}$ & $\mathbf{H}$ & $\mathbf{L}$ & $\mathbf{H}$ & $\mathbf{H}$ & $\mathbf{L}$ & $\mathbf{L}$ & - & $\mathbf{H}$ & - & - & - & $\mathbf{H}$ & $\mathbf{H}$ & $\mathbf{H}$ & $\mathbf{L}$ \\
\hline IVDDM & - & $\mathrm{H}$ & $\mathbf{L}$ & L & $\mathbf{L}$ & $\mathbf{L}$ & $\mathbf{L}$ & $\mathbf{L}$ & - & $\mathbf{H}$ & $\mathbf{L}$ & $\mathbf{L}$ & $\mathbf{H}$ & H & - & - & $\mathbf{L}$ & - & $\mathbf{H}$ & $\mathbf{H}$ & - \\
\hline
\end{tabular}

There were 8 deer in all comparisons except in April 1979-80 when there were 6.

2There was a January foraging period only in 1979-80.

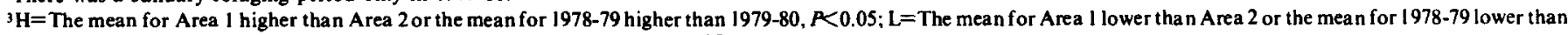
$1979-80, \mathbb{R}<0.05 ;=$ no difference between means for Areas or Years, $P>0.05$. 

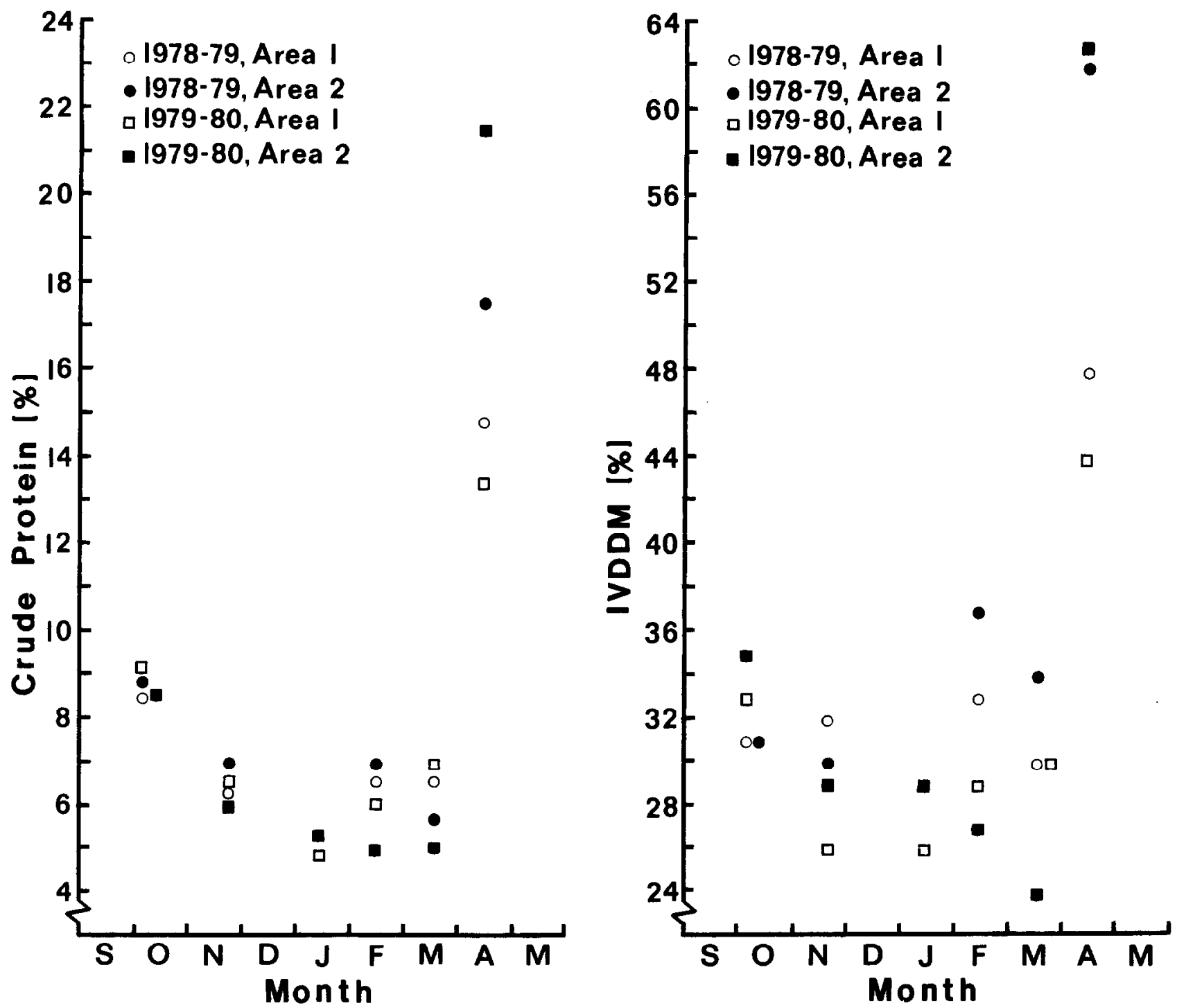

Fig. 1. Crude protein and in vitro digestible dry matter (IVDDM) content of mule deer diets on pinyon-juniper-mixed shrub (Area I) and pinyon-junipersagebrush (Area 2) winter range in Piceance Basin, Colorado, 1978-79 and 1979-80.

stood. Therefore, we can only rely on observations of food habits to determine what forages are probably most beneficial to deer welfare in any particular situation. In Piceance Basin, browse is critical to winter survival of deer because it is the most available forage in deep snow. Moreover, browse seems to have a slight nutritional advantage over forbs and grasses selected by tame deer. Forbs and grasses, however, are still important as they ease browsing pressure on shrubs and offer important nutritonal advantages during spring.

Under most winter conditions, forage quantity does not appear a major limitation to mule deer welfare within much of Piceance Basin. Therefore, habitat improvement as a mitigation alternative should stress increasing nutritional quality of existing forages during winter dormancy. However, any practice with this potential should be tested with species and conditions that exist in Piceance Basin and be applied on a large enough scale to be meaningful.

Reclaiming disturbed areas is a more complex problem as habitat must be established from bare ground-a difficult task in semi-arid environments. The aspect of reclamation this study can address is to identify native species important as winter forage for deer. Revegetation for benefit of mule deer should include a mix of trees, shrubs, forbs, and grasses to allow deer to select the most favorable nutritional regime and to help assure some species are available under all winter conditions. The 39 species listed in Tables 1 and 2 were important diet items during at least one period of winter and should therefore be given prime consideration in revegetation efforts.

Obviously, much more research is needed on how and why deer utilize available habitats for both food and cover to improve upon the above generalizations. Such information can then be used to generate and test hypotheses concerning practices for improving pinyon-juniper winter range for mule deer. Until this occurs, the best action in Piceance Basin may be no action as the Basin has consistently ranked among the top deer harvest areas in Colorado. Also, deer densities, there already exceed those measured on other large winter ranges in the state with estimates up to $20 \mathrm{deer} / \mathrm{km}{ }^{2}$ over $1,722 \mathrm{~km}^{2}$ of winter range in recent years (Bartmann, unpubl. data). Although both higher and lower deer densities occur on smaller units within the winter range, there presently is no evidence they are a direct result of forage quantity or quality differences between these areas. Until reasons for these density differences are identified, benefits of habitat improvement efforts for deer will continue to be questionable.

\section{Literature Cited}

Ammann, A.P., R.L. Cowan, C.L. Mothershead, and B.R. Baumgardt. 1973. Dry matter and energy intake in relation to digestibility in whitetailed deer. J. Wildl. Manage. 37:195-201.

A.O.A.C. 1965. Official methods of analysis (10th ed.). Association of Official Agricultural Chemists. Washington, D.C. 
Baker, D.L., and N.T. Hobbs. 1982. Composition and quality of elk summer diets in Colorado. J. Wildl. Manage. 46:694-703.

Bartmann, R.M., and S.F. Steinert. 1981. Distribution and movements of mule deer in the White River drainage, Colorado. Colo. Div. Wildl. Spec. Rep. 51.

Bartmann, R.M., and L.H. Carpenter. 1982. Effects of foraging experience on food selectivity of tame mule deer. J. Wildl. Manage. 46:813-818.

Bartmann, R.M., A.W. Alldredge, and P.H. Neil. 1982. Evaluation of winter food choices by tame mule deer. J. Wildl. Manage. 46:807-812.

Bergerud, A.T., and L. Russell. 1964. Evaluation of rumen food analysis for Newfoundland caribou. J. Wildl. Manage. 28:809-814.

Bissell, H.D., and H. Strong. 1955. The crude protein variations in the browse diet of California deer. Calif. Fish and Game 41:145-155.

Carhart, A.H. 1943. Deer-elk survey. Colo. P-R Proj. 4-R Suppl. Rep. 20p.

Cluff, L.K., B.L. Welch, J.C. Pederson, and J.D. Brotherton. 1982. Concentration of monoterpenoids in the rumen ingesta of wild mule deer. $J$. Range Manage. 35:192-194.

Cook, C.W., and L.E. Harris. 1967. Effects of supplements on nutrient intake of range forage. J. Anim. Sci. 26:934.

Cook, C.W., and L.E. Harris. 1968. Nutritive value of seasonal ranges. Utah Agr. Exp. Sta. Bull. 472.

Einarson, A.S. 1946. Crude protein determination of deer food as an applied management technique. Trans. N. Am. Wildl. Conf. 11:309-312.

Gaare, E., A. Sorensen, and R.G. White. 1977. Are rumen samples representative of the diet? Oikos 29:390-395.

Hobbs, N.T., and D.C. Bowden. 1982. Confidence intervals on food preference indices. J. Wildl. Manage. 46:505-507.

Hobbs, N.T., D.L. Baker, J.E. Ellis, and D.M. Swift. 1979. Composition and quality of elk diets during winter and summer: a preliminary analysis. p. 47-53. In: M.S. Boyce and L.D. Hayden-Wing (eds.), North American elk: ecology, behavior, and management. Univ. Wyo., Laramie. $294 \mathrm{p}$.

Hobbs, N.T., D.L. Baker, and R.B. Gill. 1983. Comparative nutritional ecology of montane ungulates during winter. J. Wildl. Manage. 47:1-16.

Hobbs, N.T., D.L. Baker, and R.B. Gill. 1983. Comparative nutritional ecology of montane ungulates during winter. J. Wildl. Manage. 47: (in press).

Holter, J.B., H.H. Hayes, and S.H.Smith. 1979. Protein requirement of yearling white-tailed deer. J. Wildl. Manage. 43:872-879.

Jobman, W.G. 1972. Consumption of juniper by deer and inhibition of rumen microorganisms by volatile oils of juniper. M.S. Thesis, Colo. State Univ., Fort Collins. 51 p.
Kufeld, R.C., O.C. Wallmo, and C. Feddema. 1973. Foods of the Rocky Mountain mule deer. U.S. Dep. Agr. Forest Serv. Res. Paper RM-111.

Mikchunas, D.G., M.I. Dyer, O.C. Wallmo, and D.E. Johnson. 1978. In vivo/in vitro relationships of Colorado mule deer forages. Colo. Div. Wildl. Spec. Rep. 43.

Nagy, J.G., H.W. Steinhoff, and G.M. Ward. 1964. Effects of essential oils of sagebrush on deer rumen microbial function. J. Wildl. Manage. 28:785790.

Norris, J.J. 1943. Botanical analyses of stomach contents as a method of determining forage consumption of range sheep. Ecol. 24:244-25I.

Pearson, H.A. 1970. Digestibility trials in vitro techniques. p. 85-92. In: H.A. Paulson, Jr., E.H. Reid, and K.W. Parker (eds.), Range and wildlife habitat evaluation-a research symposium. U.S. Dep. Agr. Forest Serv. Misc. Publ. 1147.

Regelin, W.L., R.M. Bartmann, D.W. Reichert, and P.H. Neil. 1976. The influence of supplemental feed on food habits of tamed deer. U.S. Dep. Agr. Forest Serv. Res. Note RM-316.

Robbins, C.T., P.J. Van Soest, W.W. Maut2, and A.N. Moen. 1975. Feed analysis and digestion with reference to white-tailed deer. J. Wildl. Manage. 39:67-79.

Terwilliger, C.W., Jr., C.W. Cook, and P.L. Sims. 1974. Ecosystems and their natural and artificial rehabilitation. p.67-97. In: Surface rehabilitation of land disturbances resulting from oil shale development. Tech. Rep. Series No. 1, Colo. State Univ., Fort Collins. 255 p.

Tiedeman, J.A., and C. Terwilliger, Jr. 1978. Phyto-edaphic classification of the Piceance Basin. Range Sci. Dep, Range Sci. Series No. 31. Colo. State Univ., Fort Collins. 265 p.

Tilley, J.M.A., and R.A. Terry. 1963. A two-stage technique for in vitro digestion of forage crops. J. Brit. Grassl. Soc. 18:401-411.

Voomies, K.C. 1974. A vegetation inventory and analysis of the Piceance Basin and adjacent drainages. M.A. Thesis. West. State Coll. of Colo., Gunnison. 243 p.

Wallmo, O.C., L.H. Carpenter, W.L. Regelin, R.B. Gill, and D.L. Baker. 1977. Evaluation of deer habitat on a nutritional basis. J. Range Manage. 30:122-127.

Wailmo, O.C., R.B. Gill, L.H. Carpenter, ad D.W. Reichert. 1973. Accuracy of field estimates of food habits. J. Wildl. Manage. 37:556-562.

Weir, W.C., and D.T. Torrell. 1959. Selective grazing by sheep as shown by a comparison of the chemical composition of range and pasture forage obtained by hand clipping and that collected by esophageal-fistulated sheep. J. Anim. Sci. 18:641-649. 